——ONLINE FIRST(NOT Peer-Reviewed)

Title : Techniques and methods of seismic data processing in active volcanic areas: some applications to multichannel seismic profiles (Gulf of Naples, Southern Tyrrhenian sea, Italy)

Received: 2019-03-14

Accepted: 2019-03-22

Process: 1、First trial(Field and check)

2、Peer review

3、 Editing and three trials

4. Published online

EnPress Publisher, LLC. United States 


\title{
Techniques and methods of seismic data processing in active volcanic areas: some applications to multichannel seismic profiles (Gulf of Naples, Southern Tyrrhenian sea, Italy)
}

\begin{abstract}
The techniques of seismic surveying, especially reflection seismic, considerably varied during last year's. The contribution to this variation mainly came from the oil industry, which has developed the geophysical methods for oil searching. The basic techniques of seismic exploration consist of the generation of seismic waves artificially in the ground (source) and of the measurement of the requested times to cover the source-receiver path. Seismic data processing of three multichannel seismic profiles located in the Gulf of Naples for an overall length of 150 kilometers is herein presented. The techniques of seismic processing used for the elaboration of the seismic data are up-to-date. Some of them are based on complex mathematical models, allowing obtaining good velocity analysis for the production of stacked sections, ready to be interpreted. In this paper the procedures of processing of multichannel seismic data starting from the field data are shown. Sketch diagrams of the elaboration processes applied during several phases of the whole processing have been constructed. The used software are the Promax2D (Landmark Ltd.) and the Seismic Unix (Colorado School of Mines). The steps of the seismic data processes included the pre-processing, the sorting, the velocity analysis, the normal move-out (NMO), the stacking, the band-pass filtering, the multiple removals, the predictive de-convolution and the spiking de-convolution.
\end{abstract}

\section{Keywords}

Seismic data processing; multichannel seismic profiles; Gulf of Naples; Southern Tyrrhenian sea. 


\section{Introduction}

The seismic ray path may be reconstructed through the refraction, if the times of the refracted phases are considered, or through the reflection, if the reflected phases are considered. One of the most important advantages of the seismic reflection method, if compared to the seismic reflection method is represented by the condition of velocity increase with depth. An abrupt velocity variation, both increasing and decreasing is enough to determine a reflection of the seismic waves at the discontinuity surface.

Some principles of the propagation and phases of the seismic waves are herein resumed. The, propagation of the seismic waves may be described through the Huygens-Fresnel and Fermat-Snell principles. Accordingly to the Huygens-Fresnel principle each point of the wave front may be considered as a secondary source, where the envelopment of the new wave fronts defines the location of the primary reflection during a next time, being the secondary waves active only in the direction of propagation. The wave motion happens in such a way that each point of the wave front proceeds in a direction perpendicular to it. Therefore, the wave paths may be defined as half straight lines perpendicular to the wave front, along which the perturbation proceeds.

Instead, the Fermat principle describes the path of wave ray in the inner portion of the material. Given a distribution velocity in a medium it is possible to geometrically define, using this principle, the path of the wave ray. It can be demonstrated that the reflection and refraction laws derive from the Fermat principle. The principle of superimposition follows as a consequence of the linearity of the wave equation. Based on this principle the superimposition of a certain number of waves is equal to the sum of the effects of the single components. This principle is basic in the Fourier analysis (Xu et al., 2005; Liu et al., 2011; Liu and Fomel, 2013; Wang et al., 2014).

The velocity and the direction of propagation of the different types of waves vary depending on the physical properties and the dimensions of the crossed medium. The waves travel with a constant velocity along rectilinear paths in an ideal infinite medium, homogeneous, isotropic and perfectly elastic. The Earth is supposed to be stratified, with layers having a different thickness and variable physical characteristics (velocity, density) and not perfectly elastic neither isotropic. Perhaps, variations both in the direction and in the velocity propagation and in the amplitude and phase characteristics occur in the earth. Accordingly to the rules of the geometrical optics when a wave path encounters a surface of separation between two media supposed to be homogeneous but having different physical characteristics (in particular the velocity), a part of the energy is reflected and a part is transmitted in the second medium. The phenomenon is called refraction if during the wave transmission a change of direction is observed. The direction of the reflected path is determined through the law of reflection.

The seismic waves propagate in the inner of the earth with velocity, frequency and amplitude depending on the elastic properties of the rocks. In a medium crossed from a wave train two types of waves propagate: the longitudinal $\mathrm{P}$ waves, vibrating in the same direction of the propagation and the transversal $\mathrm{S}$ waves, having a vibration plan perpendicular to the propagation one.

For the $\mathrm{P}$ waves the wave equation is of the following type (Sheriff and Geldart, 1995):

$1 / \alpha^{2} \cdot \partial^{2} \Delta / \partial t^{2}=\Delta^{2} \Delta$

(1)

where the velocity $\alpha$ is given from the following equation: 
$\alpha^{2}=\lambda+2 \mu / \rho$

(2)

For the $\mathrm{S}$ waves the wave equation is of the following type (Sheriff and Geldart, 1995):

$1 / \beta^{2} \cdot \partial^{2} \partial_{x} / \partial t^{2}=\Delta^{\partial} / \partial_{x}$

where the velocity $\beta$ is given from the following equation:

$\beta^{2}=\mu / \rho$

(4)

\section{The reflection and transmission coefficients}

The problem of the determination of the equations which put in relationships the coefficients of amplitude of the incident, reflected and refracted waves has been studied by Knott (1899) and Zoeppritz (1919). Accordingly to Zoeppritz (1919) the equations of partition of the amplitudes at a seismic interface may be expressed as:

$A_{1} \cos \partial_{1}-B_{1} \sin \lambda_{1}+A_{2} \cos \partial_{2}+B_{2} \sin \lambda_{2}=A_{0} \cos \partial_{1}$

(5)

$A_{1} \sin \partial_{1}-B_{1} \cos \lambda_{1}+A_{2} \sin \partial_{2}+B_{2} \cos \lambda_{2}=A_{0} \sin \partial_{1}$

(6)

$A_{1} Z_{1} \cos 2 \lambda_{1}-B_{1} W_{1} \sin 2 \lambda_{1}+A_{2} Z_{2} \cos 2 \partial_{2}+B_{2} W_{2} \sin 2 \lambda_{2}=A_{0} Z_{1} \cos 2 \lambda_{1}$

$A_{1} \gamma_{1} W_{1} \sin 2 \vartheta_{1}-B_{1} W_{1} \cos 2 \lambda_{1}+A_{2} \gamma_{2} Z_{2} \sin 2 \partial_{2}+B_{2} W_{2} \cos 2 \lambda_{2}=A_{0} \gamma_{1} Z_{1} \sin 2 \vartheta_{1}$

(8)

where $\quad A_{0} \quad A_{1} \quad A_{2} \quad$ are the amplitudes of the $\mathrm{P}$ waves, respectively incident, reflected and refracted;

$\vartheta_{1}$ and $\vartheta_{2}$ are the reflection and refraction angles;

$B_{1}$ and $B_{2}$ are the amplitudes of the $\mathrm{S}$ waves, both reflected and refracted;

$Z_{1}$ and $Z_{2}$ are the acoustic impedances.

In order to apply these equations to an interface we must know the density and the velocity of each medium to determine the parameters $\gamma_{1}, Z_{1}$ and $W_{1}$. Knowing $A_{0}$ and $\vartheta_{1}$ from the Snell law we calculate $\vartheta_{2}, \lambda_{1}$ and $\lambda_{2}$. The coefficients expressing the amplitudes $A_{1} \quad A_{2}, B_{1}, B_{2}$ remain to be calculated. The reflection gives rise each time that there is an abrupt contrast of acoustic impedance. The widest is the contrast of acoustic impedance between the media, the strongest is the reflection. These variations of acoustic impedance correspond to geologic variations including the lithology, the facies and corresponding depositional environments, the porosity, the fluid content and the strata intervals.

In the case of the normal incidence of a $\mathrm{P}$ wave (hypothesis which may be applied to the seismic reflection method) the Zoeppritz equations may be reduced to two equations: 
$A_{1}+A_{2}=A_{0}$

(9)

$Z_{1} A_{1}+Z_{2} A_{2}=Z_{1} A_{0}$

(10)

By solving the equations with respect to $A_{1}$ and $A_{2}$ we obtain:

$\frac{A_{1}}{A_{0}}=\frac{Z_{1-} Z_{2}}{Z_{2+} Z_{1}}$

(11)

$\frac{A_{2}}{A_{0}}=\frac{2 Z_{1}}{Z_{2+} Z_{1 i}}$

\section{Geometry of the seismic events}

The ray theory allows for showing in a simple way, accordingly to the criteria of the geometrical optics, the relationships between the times of arrival of the reflected and refracted waves and the location of the discontinuities bounding the geological strata. In the two-dimensional cases, with parallel and plan strata we consider a layer having a thickness $h$ on a homogeneous half-space, being $\mathrm{x}$ the distance between the $\mathrm{S}$ energy source and the $\mathrm{r}(\mathrm{x})$ receiver. We discuss here the travel times of the direct, reflected and refracted phases. The direct wave will reach the $\mathrm{r}(\mathrm{x})$ receiver after a time:

$t_{d}=\frac{x}{v_{1}}$

which is the equation of a straight line passing for the origin.

The refracted phase covers the path (racS) during the time:

$t_{r}^{2}=\frac{x}{v_{2}}+\frac{2 H}{V_{1}} \cos \left(i_{c}\right)$

representing the equation of a straight line which doesn't cross the origin and having an angular coefficient $1 / v_{2}$.

The reflected phase which will travel in the pathway $(\mathrm{rbS})$ will reach the receiver after a time: 
$t_{\text {rifl }}^{2}=\frac{1}{v_{1}}\left(\frac{x}{2}\right)^{2}+\frac{H^{2}}{V_{1}} ; t_{\text {rifl }}=\frac{1}{v_{1}} \sqrt{\left(\frac{x}{2}\right)^{2}}+\sqrt{H^{2}}$

which is the equation of an hyperbola symmetrical with respect to the axes $\mathrm{x}$ and $\mathrm{t}$, having as asymptote some straight lines passing for the origin with angular coefficients $+/-\frac{1}{v_{1}}$. The asymptotes coincide with the direct wave.

The depth of the reflecting interface is individuated by the time of arrival of the reflected wave in the source point (offset 0 ).

$t_{0}=\frac{2 \cdot H_{1}}{V_{1}}$

If we consider a point on the reflection hyperbola corresponding to the time $t_{1}$ and carrying out several calculations (herein not reported for simplicity), we obtain the following equation:

$\Delta \mathrm{T}(\mathrm{x})=\frac{x^{2}}{2 V_{1}^{2}} \cdot \frac{1}{v_{0}}$

$\Delta \mathrm{T}(\mathrm{x})$ represents the Normal Move Out (NMO). The velocity $\mathrm{V}_{1}$ represents the NMO velocity, necessary for the NMO correction on the traces related to the considered CDP (Common Depth Point).

\section{Materials and Methods}

From the 4 to the 28 June 1999 a digital seismic survey has been carried out aimed at investigating the Southern Tyrrhenian sea and in particular, the Naples Bay (Aiello et al., 2011a; 2011b). More than 2400 kilometers of seismic lines have been recorded. The employed instruments and techniques have allowed for obtaining good quality seismic data, especially in active volcanic areas where the occurrence of pyroclastic levels and buried volcanic edifices produce a strong scattering of the energy of the elastic waves. Two Airguns have been employed coupled with a 48 channel seismic streamer and a seismic acquisition system (Geometrics Inc.). The Airgun is a seismic source adopted in the marine environment based on the release of pressured air in the surroundings (Di Fiore et al., 2006; ISPRA, 2017). The aims of the research using the Airgun seismic source include the climatic changes, the planning of the use of the marine environment, the natural hazard in the marine environment, the security of the industrial and strategic structures, the natural seismicity and the seismicity induced by the human activities and the geothermal and natural resources. In this paper the elaboration of three multichannel profiles is presented, located in the Gulf of Naples. The acquisition parameters are shown in Table 1. 


\section{Results}

The used techniques of seismic data processing are based on complex mathematical models, allowing for obtaining a good multiple attenuation (especially sea bottom multiples) and good velocity analyses to produce stacked sections for the geologic interpretation. The procedures of data processing are herein shown starting from the field data through the construction of sketch diagrams of flux elaboration. Promax 2D and Seismic Unix have been used.

Promax 2D (Landmark Graphic Corporation, 1998) has presented a general workflow of seismic data processing (Table 2). The steps distinguished in this flux diagram include the assignment of the geometry, the editing of the seismic traces, the selection of the parameters, the elevation statics, the refraction statics, the brute stack, the velocity analysis, the residual statics, the dip moveout (DMO), the post-stack signal enhancement and the post-stack migration, allowing for the velocity modeling. The geometry assignment has been designed in order to create the database files of the Promax 2D program and to load the header information into the trace headers of the Promax 2D data. After assigning the geometry map, the land geometry is determined and consequently, the shot gathers are viewed. The geometry is loaded in the spreadsheet and in the database and the database attributes are viewed. After, the geometry is loaded to the trace headers and the graphical geometry is determined. The geometry assignment has been designed to create the Promax database files and to load the header information into the trace headers of the seismic data processed in the Promax. The sequence of steps or flows mainly depends upon the available information. The steps necessary to assign the geometry to a seismic line are all required, if all the information is present in the trace headers of the input dataset. This approach may be useful in order to re-process the seismic data. The basic Promax framework is closely related to the UNIX directory structure and the manipulation of the various components of the line database is of critical importance in order to smooth. The main steps are represented by the understanding of where and how the data files have been stored, where the menus and the program have been stored and how the data pass through a Promax flow.

Seismic Unix is an open source program, which is supported by the Center of the Wave Phenomena (CWP) of the Colorado School of Mines. This software has many processes which are needed in the geophysical processing, making possible the manipulation and the creation of the seismograms and their conversion from the SEG Y format, which is the standard format of seismic acquisition and the SU standard file. The first main program is represented by the data compression (Table 3). The second one is represented by the editing, the sorting and the manipulation (Table 4). Other programs are represented by filtering, transforms and attributes and Gain, NMO, Stack and Standard Processes.

A flux of elaboration of seismic data has been defined for the multichannel seismic data of the Gulf of Naples (Fig. 1). The processing of the seismic data is herein described. During the pre-processing phase the acquisition geometry has been assigned to the seismic data using the acquisition parameters reported in Table 1. All the seismic traces considered to be too noisy (trace kill) have been eliminated. The muting of the first arrivals is an operation aimed at eliminating all the seismic noise above the first arrivals and the un-desired spikes in the seismograms. In the case of the spikes zones of the seismograms are put to zero, on which a data extrapolation allowing to obtain a continuity of amplitude in the seismograms and, in such a case, to avoid possible mistakes, when the operations between the seismic traces are performed. The application of the gain is the 
compensation of the signal of decay, due to the phenomena of absorption, scattering and decay of the amplitude and is necessary to restore the lost signal and to obtain similar levels of amplitude in the whole seismic data. The corresponding process is the Automatic Gain Control (AGC). This technique varies the gain of the seismic traces as a function of the amplitude occurring in a time window, whose extension has been determined through a length operator, defined during the data elaboration. The AGC shifts this time window downwards, sample by sample, by calculating a scaling factor for each location. This scaling factor may be equal to the reverse of the medium, of the median and of the RMS of the amplitude of the signal enclosed in the window. In the processed lines, the choose window for the AGC application was $2000 \mathrm{msec}$; to this value we arrived after several tests on the seismic data.

It is important also speak about the sorting. One of the fundamental steps which have make the exploration seismic to be the reference method in the field of the geophysical techniques, both industrial and scientific is the acquisition technique with a multiple coverage. This technique allows to obtain a redundant information for the same point surveyed at depths.

Accordingly to the simple laws of the reflection, the reflecting point is put on the vertical of the median point source-receiver. Using different scattered configurations it is possible to record a certain number of shots, in such a way that a median point (represented by the reflection point) is common to all the source-receiver locations. In this way we obtain a set of signals of reflections which are all reflected by the same point at depths (Common Depth Point or CDP).

The method of multichannel reflection seismic having a multiple coverage has been introduced as a routine in the field of data acquisition during the sixties (Mayne, 1962; 1967). A model has been constructed. Based on this model the coordinates are $y, h$ (median point, offset), defined in terms of $\mathrm{s}, \mathrm{g}$ as it follows:

$\mathrm{y}=(\mathrm{g}+\mathrm{s}) / 2$

$\mathrm{h}=(\mathrm{g}-\mathrm{s}) / 2$

Seismic traces may be ordered in groups of various type, such as the Common Shot Gather (field recording), the Common Receiver Gather (groups of traces related to the same acquisition channel), Common Depth Point Gather (generally called the CMP-gather), Common Offset Gather (re-order of the seismic traces based on the source-receiver distance), CMP-Stacked section (section ready to be interpreted).

The velocity analysis also needs to be clarified. When the data have been ordered in CDP (Common Depth Point), the velocity analysis has been performed on the same data aimed at producing the first stacked section. The same velocity analysis has been repeated after the application of new processes of data elaboration, trying to understand if the same processes produce or not a significant improvement of the seismic signal.

The velocity analysis has a fundamental importance to apply the Normal Move Out (NMO) correction (Yilmaz, 1987):

$\sqrt{t_{0}^{2}}+\sqrt{\frac{x^{2}}{v^{2}}}-t_{0}$

The velocity analysis is necessary to make horizontal the reflection hyperbola before carrying out the sum (stack) of the seismic traces. Through the velocity analysis information is obtained on the 
velocities of the seismic reflectors occurring on the seismic sections. Different techniques to perform the velocity analysis exist. In the most of cases the Constant Velocity Stack (CVS) has been adopted, then compared with the semblance. The CVS consists of the selection of a portion of datum, from a minimum of $5 \mathrm{CMP}$ to a maximum of $25 \mathrm{CMP}$ and in the generation of several stack panels. Each panel is obtained using velocity values varying between the lowest and the highest velocity value attained based on a first datum analysis. The function velocity/depth has been obtained individuating on several panels, corrected for the NMO, the best corrected zones, based on the velocity indicating the panel. The examples of the velocity values obtained with this technique have been then revised using the velocity spectra (semblance), in which a CMP gather is considered. After carrying out the velocity analysis it is necessary to correct the reflection hyperbola, clarifying the sense of the NMO correction. The simplest case is the horizontal reflector. In this case, if we report on a Cartesian diagram $x-t$ the times of arrival of the reflected wave for each receiver we will obtain a hyperbola. The OM straight line is the graphic of the times of arrival of the direct wave, whose path is $S-R$ and is characterized by a velocity $t_{d}=x / V$.

In order to calculate the path travelled by the reflected wave to reach the geophone $\mathrm{R}$ it is necessary to project the point I below the seismic reflector to a distance equal to the distance between the source (S) and the seismic reflector (h) and then to join I and R. For a geometrical construction we will have that the space travelled from the wave $(\mathrm{SC}+\mathrm{CR})$ is equal to the space obtained through our construction. The hyperbola constructed through the times of arrival of the reflected wave is a consequence of the fact that the times for farthest geophone are greater. The difference between the times of arrival of the wave to two distant geophone is defined as moveout. $\Delta t$. Then the move out represents the delay accumulated from the wave front of reflection in reaching the farthest receivers. In the particular case of a receiver located at the source we will calculate the following equation:

$\Delta \mathrm{t}_{\mathrm{NMO}}=\mathrm{t}_{\mathrm{x}}-\mathrm{t}_{0}$

where $\Delta \mathrm{t}_{\mathrm{NMO}}$ is defined as the Normal Move Out (NMO), $\mathrm{t}_{\mathrm{x}}$ is the time of arrival of the wave at the receiver in $\mathrm{R}$, while $\mathrm{t}_{0}$ is the time recorded from the receiver to the source.

Re-writing the equation (21) as a function of the velocity and of the distance and expressing $t_{x}$ as a function of $\mathrm{t}_{0}$ we will have:

$\Delta \mathrm{t}_{\mathrm{NMO}}$

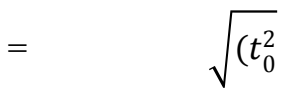

$\sqrt{\left(t_{0}^{2}\right.}$

$+\sqrt{\frac{x^{2}}{v^{2}}}$

)

$\mathrm{t}_{0}$

By examining the equation (22) we noted that the NMO increases with the offset $\mathrm{x}$, representing the source-receiver distance and is reversely proportional to the velocity. The curvature of the traveltime curve of the reflected waves rapidly increases considering receivers farther and farther, but decreases with the increase of the recording time. The NMO correction is applied before carrying the sum (stack) of the traces in order to eliminate the curvature which prevents to correctly locate the reflector. The correction is carried out by inserting a velocity value calculated from the velocity analysis in the corresponding formula. If this value is right we will obtain that the traces will be correctly aligned. For higher velocity values we will obtain a minor correction value and the traces will show a residual curvature. On the other side, if the velocity value is too low we will have an 
over-correction of the times of arrival and then the traces will show an opposite curvature.

Speaking on the stacking is also important. The stacked section generally represents the final product of the elaboration of the seismic data. It is composed of traces representing the in-phase sum (stack) of the traces coming from the same CDP. The stacking allows for increasing the signal-to-noise ratio $(\mathrm{S} / \mathrm{N})$ reducing the casual noise included in the data. During the stacking the coherent signal will increase its amplitude, for constructive interference, of a factor equivalent to the data coverage, while the casual noise will add to other noise, only increasing its amplitude.

The band-pass filtering needs also to be clarified. In many applications, such as the analysis of the seismic signals and more in general, of the geophysical data, given a signal $f(t)$ and assuming that $t$ is a continuous variable we are interested to its content in frequency. The continuous Fourier transform is defined as:

$\mathrm{f}(\omega)=\int_{-\infty}^{+\infty} f(t) \cdot e^{(i \omega t)} \mathrm{dt}$

The Fourier analysis is performed on the seismic traces in order to identify the frequency content (Bracewell, 1965) of the seismic signal in several seismograms and eventually, to apply a band pass filter, which allows to enhance the interesting frequencies. The filtering consists of the modification of a time series through the application of another time filtered series, allowing for the enhancement of the frequencies of interest. The filtering consists of the modification of a time series through the application of another time-filter series, opportunely constructed. The application of a filter consists of the convolution of the filter itself with the seismogram. A generic bandpass filter has an answer in amplitude equal to the frequencies ranging between $\mathrm{p}$ and $\mathrm{q}$. This interval is called band-passing. These values of frequency are given in correspondence with the points in which the A amplitude is of three decibel lower than the band pass.

The applied filter consists of inserting some frequency values individuating the vertexes of a trapeze and a parameter which controls the slope of the sides of the trapeze. This last parameter, expressed in decibel/octava is important to know how much are reduced the frequencies external to ties e passing band, after the filter application. The choice of the filter has been done after analyzing the content in frequencies of the data, in order to individuate the frequency interval in which the useful signal was concentrated. The chosen filter resulted to be quite conservative $(0-20-50-70 \mathrm{~Hz})$, allowing, in any case to eliminate the high-frequency noise occurring in the data.

The techniques of multiple removal have also been applied. The multiple reflections verify when the seismic signal is reflected more times from the discontinuities located in the inner of the earth before arriving to the sensors. Since the amplitude of the reflections is proportional to the reflection coefficients of each seismic reflector, only the strongest contrasts of acoustic impedance generate signals strong enough to be recognized as events. Short-path and long-path multiples may be distinguished.

The reference event for this classification is the primary reflection. The effect of the short-path multiple translates as a deformation of the primary wave, since the two reflections are one near to each other and it can be individuated with difficulties without a direct analysis of the wave shape of the source.

The long-path multiple can be individuated as a distinct event on the stacked sections. Several techniques exist for the suppression of the multiples, whose knowledge is based on one of the 
following characteristics of the multiple reflections: 1) difference of moveout between primary and multiple reflections (discriminant of velocity); 2) difference of inclination between the primary and the multiple events in the CDP stacked sections; 3) difference of the frequency content between the two types of signals; 4) periodicity of the multiples. In the study case the procedure of attenuation of the multiples herein applied are the stacking and the predictive deconvolution. We have discriminated the moveout between the primary and the multiple reflections through the stacking and defining a correct function of velocity between the primary reflections. In this case the coherent noise under-correct has been attenuated in the sum not in phase of the related events (Marr and Zagst, 1967). The efficacy of the stacking improves with the increase of the coverage and of the maximum offset, increasing the number of traces to be added in the CMP gather. The predictive deconvolution has been carried out on the seismic data aimed at eliminating or further reducing the multiple signal which has characterized the same seismic sections, also if the same operation has not always produced significant improvements. In each case, the occurrence of multiples in the seismic signal has been useful during the phase of geologic interpretation for a better definition of the main seismic reflectors and a comparison of the stratigraphic relationships between the same reflectors.

The deconvolution was a main step in the elaboration of the seismic signal, allowing to recover the high frequencies, to attenuate the multiples and to reconstruct the wave shape. This process is an operation in the time domain, whose aim was the removal of the effects of the convolution on the recorded data. The deconvolution simplifies the seismic wave of the source $w(t)$ from the recorded traces in order to reconstruct the function of the earth's reflectivity e $(\mathrm{t})$, through which the wave is convoluted:

$s(t)=e(t) * w(t)+n(t)$

and consequently, to increase the time resolution of the data. In practice, it consists of the convolution of the seismogram with a reverse filter (Wiener filter). The principle of the deconvolution is to remove the effects of a previous filter, the earth, which reduce the high frequencies. The main difficulty in performing this operation consists of the construction of the reverse filter, since the properties $\mathrm{w}(\mathrm{t})$ are unknown.

The deconvolution may be distinguished as: 1) spiking deconvolution, aimed at removing the effects of the wave from the source of the seismogram, controlling a widening and a flattening of the spectrum of the frequencies; 2) predictive deconvolution, aimed at predicting and eliminating the reverberations which are included in the seismic signal, such as for instance the multiples.

The spiking deconvolution is theoretically defined starting from the convolution model, in which the seismogram is expressed from:

$s(t)=e(t) * w(t)$

in which $\mathrm{s}(\mathrm{t})$ is the recorded seismogram, $w(t)$ is the seismic wave from the source and $\mathrm{e}(\mathrm{t})$ is the impulsive answer of the earth. The earth's reflectivity e $(t)$ may be calculated applying the Wiener filter $\mathrm{f}(\mathrm{t})$, which modifies the shape of the wave by compressing it and approaching it to the Dirac Delta function:

$e(t)=f(t) * s(t)$ 
where the function of the Dirac Delta $\delta$ may be defined as an impulsive function which assumes the infinite value for $\mathrm{t}=0$ in each other point:

$\begin{aligned} \delta(t)= & \{\infty, t=0 \\ & \{0, t \neq 0\end{aligned}$

After consequent calculations we calculate the filter $\mathrm{f}(\mathrm{t})$ :

$\mathrm{f}(\mathrm{t})=\delta(\mathrm{t}) * \frac{1}{w(t)}$

By resuming we have obtained, applying the filter to the seismogram, an impulsive function and the corresponding equation (28) establishes that the filter is the reverse of the seismogram.

Regarding the predictive deconvolution, if we consider our seismogram $x(t)$, the aim of this deconvolution is to predict the value of the function $\mathrm{x}(\mathrm{t})$ at the time $(\mathrm{t}+\alpha)$, being $\alpha$ the distance of prediction. The bases of the predictive deconvolution are the same of the spiking decomvolution. The main difference consists of the construction of the reverse filter and in particular, of the choice of the parameters.

The parameters to know in order to construct the filter necessary to the deconvolution are: 1) the window of application, which is the window of the datum on which the auto-correlation has been applied; 2) the distance of prediction or gap, which is the part of the wave which we want to preserve. It represents the delay before the multiple or the reverberation to be eliminated.

Both the types of deconvolution have been applied to the three processed profiles. Fig. 2 shows an example of spiking deconvolution. In the upper seismic sections the seismic signal appears to be more spike (compressed) and consequently, it is simpler to define the different reflectors during the geologic interpretation.

\section{Discussion}

Techniques of seismic data processing in active volcanic areas and in particular, in the Gulf of Naples have been previously applied. Their results may be compared with the obtained data (Bruno et al., 2002; 2003; Zollo et al., 2003; 2008; Judenherc and Zollo, 2004; Bruno et al., 2018). Since the Campania volcanism develops near the sea, the geophysical study of the marine environment improves the comprehension of the tectonic evolution and of the origin of the volcanism in the studied area. Multichannel seismic lines have been re-processed in order to understand the development of volcanism in the area (Bruno et al., 2002). The re-processing of the seismic lines was aimed at the reduction of the random noise occurring in the seismic data, at the removal of the coherent events, at the reduction of the spatial aliasing, at the improvement of the seismic resolution of the data and at the re-positioning of the seismic reflectors through dip-moveout and post-stack time migration. The obtained results have shown that NE-SW trending structural strikes prevail in the Gulf of Naples, while NW-SE trending structural strikes have been observed in the Gulf of Pozzuoli (Bruno et al., 2003). A main NE-SW trending normal fault, namely the Magnaghi-Sebeto line has crossed several volcanic banks. This fault separates the Naples bay into two main sectors, 


\section{EnPress}

having different geological, geophysical and petrochemical characteristics. The structural setting has evidenced that oblique extension occurs or alternatively, a transfer zone characterized by NW-SE trending fault systems (Bruno et al., 2003).

Zollo et al. (2003) have performed a wide seismic survey on September 2001 in the Naples and Pozzuoli bays in order to obtain three-dimensional images of the shallow structure of the Phlegrean caldera. The tomographic inversion of 77.000 first $\mathrm{P}$ arrival times has been carried out using the tomographic technique of Benz et al. (1996). This tomography has allowed to recognize the buried margin of the Phlegrean caldera, ranging at depths between 800 and $2000 \mathrm{~m}$. Based on the calibration with deep borehole data the buried margin of the Phlegrean caldera is composed of lavas and tuffs. The carbonate acoustic basement has also been recognized beneath the caldera, ranging to depths of about $4 \mathrm{~km}$ (Zollo et al., 2003). Judenherc and Zollo (2004) have discussed the geophysical data recorded during the Serapis experiment of active seismics, which has covered the whole Naples Bay, focusing on the Gulf of Pozzuoli. 3D velocity structure has been computed, including a small-scale high resolution tomographic model of the Phlegrean caldera. Velocity and gravity interpretation has been calibrated with stratigraphic information coming from deep geothermal wells in order to map the lithological units at depths. In the proposed model of the Phlegrean caldera, based on tomographic inversion and mainly related with the carbonate acoustic basement, located below the caldera, no magma chamber exist in the first 6 kilometers below the Naples Bay. In the proposed model the tectonic lineaments have controlled the feeding system of the caldera, especially NE-SW trending normal faults related to the extensional tectonics of Southern Apennines. This model has been later contradicted in the paper of De Natale et al. (2016), showing new data on the caldera structure based on a 501 deep hole located westwards of the Naples town. New stratigraphic and ${ }^{40} \mathrm{Ar} /{ }^{39} \mathrm{Ar}$ geochronological data have allowed for the determination of the age of the intra-calderic deposits. The obtained results have suggested a reduction of the area affected by the caldera collapse, excluding the area covered by the Naples town, a small volume of the volcanic deposits infilling the caldera and the needs of re-assessment of the collapse amounts and mechanisms, which are related to larger eruptions. In particular, the lithological, mineralogical and micropaleontological analyses of cuttings and cores coming from the well have shown several volcanic sequences, described in detail in Mormone et al. (2015). The upper sequence (up to a depth of $36 \mathrm{~m}$ ) consists of subaerial pyroclastic deposits, representing the Agnano-Monte Spina tephra (De Vita et al., 1999). The underlying sequence is composed of sponge spicules and diatoms-bearing deposits, underlain, in turn, by obsidian and vescicular scoriae. The remaining underlying sequence is represented by greenish tuffs overlying a gray tuff, located at the bottom of the hole. At $439 \mathrm{~m}$ of depth gray-green tuffs exist, altered by clay minerals (Mormone et al., 2015; De Natale et al., 2016). The ${ }^{40} \mathrm{Ar} /{ }^{39} \mathrm{Ar}$ geochronological data have evidenced ages ranging between $47.5 \mathrm{ka}$ and $16.9 \mathrm{ka}$, allowing to detail the collapse features and the volcanological evolution. The scoria deposits occurring to a depth of $191 \mathrm{~m}$ have been related to the NYT outcrops located at the Posillipo hill, one kilometer eastwards of the drill site and overlapping the Trentaremi volcanic sequence (Scarpati et al., 2013). It is hypothesized that the base of the NYT within the caldera is not more than $250 \mathrm{~m}$ deep, while at the Posillipo cliff is located at the sea level. The textural and mineralogical features have suggested that the Campanian Ignimbrite deposits are represented by the stratigraphic interval drilled between 265 and $450 \mathrm{~m}$ of depth. Moreover, the information coming from age and microfossils has been merged with the local sea level curve (Antonioli et al., 2004). This merged information has allowed for obtaining indirect evaluation of 
the rate of NYT and CI collapses, taking as a reference point the base of the NYT sequence cropping out along the western side of the Posillipo hill. By subtracting the amount of the NYT collapse from the sea level fall of $350 \mathrm{~m}$, a collapse of $100 \mathrm{~m}$ related to the CI can be evaluated. The sediments overlying the NYT are similar to the marine sequence cropping out at the La Starza marine terrace (Gulf of Pozzuoli; Pescatore et al., 1984). The model of De Natale et al. (2016) has been later updated by Torrente and Milia (2018), which have demonstrated that the stratigraphic data of the deep borehole are consistent with a model of fault-controlled ignimbritic emissions and are inconsistent with a caldera model. Firstly, the stratigraphic data of a single well are not enough to establish that the caldera does not occur in the city of Naples. Accordingly to Bellucci et al. (2006) the stratigraphic succession of the city of Naples is formed by Middle Pleistocene marine sediments, pre-CI tuffs, CI tuffs and NYT deposits. Several points of discussion exclude the occurrence of a caldera in the Naples city such as the thickness of the CI in the study area reaches moderate values and the tuffs below the CI deposits occur to maximum depths of $200 \mathrm{~m}$ (Torrente and Milia, 2018). Moreover, at the Naples hill (S. Martino-Vomero) the volcanic deposits of the Campanian Ignimbrite overlie a previous volcanic relief instead of a caldera depression. The lacking of wide caldera collapses has been confirmed by the depth of the Middle Pleistocene marine deposits (about $300 \mathrm{~m}$ ) and by the occurrence of NE-SW trending normal faults in the Naples Bay.

The multivariate interpretation of geophysical experiments at the Solfatara volcano has demonstrated the fruitful use of different methods for geophysical imaging, highly sensitive to physical parameters of rocks and fluids (Bruno et al., 2018). Exploratory Data Analysis (EDA) techniques have been used to interpret seismic and electric data recorded along two perpendicular profiles located in the crater of the Solfatara volcano. The clustering methods have been applied to two multivariate datasets, including the trace attributes, the P-wave velocity and the electrical resistivity. The patterns and the structure of the Solfatara volcano have been evidenced not only evident based on the visual analysis in the order to better understand the distribution of faults and fractures (Bruno et al., 2018).

A map of the structural setting of the carbonate basement in the Gulf of Naples has been constructed based on the interpreted data (Fig. 3). Onshore and offshore data of the Naples area have also been considered. The structure of the carbonatic basement in the Gulf of Naples is characterized by a NW-dipping monocline. Some local geological structures are superimposed to the monocline. The first one is a palaeo-graben of the Mesozoic limestones, located in the north-eastern Naples Bay. The carbonate monocline is downthrown by NE-SW trending normal faults, controlling the sinking of the carbonate acoustic basement to the north of the Sorrento Peninsula.

\section{Conclusion}

The techniques and the methods of seismic data processing have been applied to the active volcanic area of the Gulf of Naples based on the elaboration of multichannel seismic data. The Naples and Pozzuoli bays host active volcanic areas during recent geological times. Being densely inhabited and surrounding the Vesuvius volcano and the Phlegrean Fields volcanic complex, they have prompted different seismic experiments, especially on the Vesuvius volcano (Zollo et al., 1998; 2002; Bertrand et al., 2000; De Franco et al., 2000; De Matteis et al., 2000; Scarpa et al., 2000; Auger et al., 2001; Lomax et al., 2001). The processing of the seismic data, shown in this paper has been carried out through specific software. The starting phase consisted of the quality control of the seismic data and of the assignment of the field geometry. The editing of the seismic traces was 


\section{EnPreŝs}

finalized to survey the seismic traces and the spikes, which could induce problems with the Fast Fourier Transform (FFT). The top muting has allowed for the elimination of the seismic signal located above the first arrivals of the seismic traces. The Automatic Gain Control (AGC) has improved the normalization of the seismic traces. Moreover, the data processing was aimed at reducing the casual noise occurring in the seismic data and at improving the resolution of the seismic wavelet with algorithms of deconvolution and spiking. The velocity analysis has been carried out to remove the move-out on the CDP families, with the aim to define the velocity of the different seismic reflectors and to produce the final seismic section. A post-stack deconvolution has been applied to the data in order to remove the multiple arrivals. A bandpass filter has been applied after the deconvolution to improve the involved signal in the seismic sections. The seismic interpretation has been carried out accordingly to the seismo-stratigraphic criteria and taking into account both the multiples and the seismic noise.

\section{Ethics Statement}

I state that the ethics of this manuscript is correct. 


\section{References}

Aiello G., Marsella E., Cicchella A. G., Di Fiore V. (2011a) New insights on morphostructures and seismic stratigraphy along the Campania continental margin (Southern Italy) based on deep multichannel seismic profiles. Rendiconti Lincei, 22 (4), 349-373.

Aiello G. Cicchella A. G., Di Fiore V., Marsella E. (2011b) New seismo-stratigraphic data of the Volturno Basin (northern Campania, Tyrrhenian margin, southern Italy): implications for tectonostratigraphy of the Campania and Latium sedimentary basins. Annals of Geophysics, 54 (3), 265-283.

Auger E., Gasparini P., Virieux J., Zollo A. (2001) Seismic evidence of an extended magmatic sill under Mt. Vesuvius. Science, 294, 1510-1512.

Antonioli F., Bard E., Potter E.K., Silenzi S., Improta S. (2004) 215-ka history of sea-level oscillations from marine and continental layers in Argentarola cave speleothems (Italy). Global and Planetary Change, 43, 57-78.

Bellucci F., Milia A., Rolandi G., Torrente M.M. (2006) Structural control on the Upper Pleistocene ignimbrite eruptions in the Neapolitan area (Italy): Volcanotectonic faults versus caldera faults. In B. De Vivo (Ed.), Volcanism in the Campania Plain: Vesuvius, Campi Flegrei and Ignimbrites. Developments in Volcanology, 9, 163-180, Springer.

Benz H.M., Chouet B., Dawson P.B., Lahr J.C., Page R.A., Hole J.A. (1996) Three-dimensional P and S wave velocity structure of Redoubt volcano, Alaska. Journal of Geophysical Research, 101, 8111-8182.

Bertrand E., Deschamps A., Virieux J. (2000) Crustal structure deduced from receiver functions via single-scattering migration. In: Capuano P., Gasparini P., Zollo A., Virieux J., Casale R., Yeroyanni M. (Eds.) The Internal Structure of Mt. Vesuvius, pp. 117-144.

Bracewell R.N. (1965) The Fourier Transform and its application. McGraw-Hill Book Co.

Bruno P.P.G., Di Fiore V., Rapolla A. (2002) Seismic reflection data processing in active volcanic areas: an application to Campi Flegrei and Somma Vesuvius offshore (Southern Italy). Annals of Geophysics, 45 (6), 753-768.

Bruno P.P.G., Rapolla A., Di Fiore V. (2003)Structural setting of the Bay of Naples (Italy) seismic reflection data: implications for Campanian volcanism. Tectonophysics, 372, 193-213.

Bruno P.P.G., Berardinelli S., Maraio S. (2018) Multivariate interpretation of co-located geophysical experiments at Solfatara Volcano (Campi Flegrei, Italy) using Exploratory Data Analysis techniques. Miscellanea INGV, Int. Cong. “Cities on Volcanoes”, Naples, September 2018, p. 85.

De Franco R., Tondi R., Caielli G., Biella G., Corsi A. (2000) 2D seismic modelling and 2D seismo-gravity sequential integrated inversion along TomoVes profiles. In: Capuano P., Gasparini P., Zollo A., Virieux J., Casale R., Yeroyanni M. (Eds.) The Internal Structure of Mt. Vesuvius, pp. 105-116.

De Matteis R., Latorre D., Zollo A., Virieux J. (2000) 1-D P-velocity Models of Mt. Vesuvius volcano from the inversion of TomoVes96 First Arrival Time Data. Pure and Applied Geophysics, 157, 1643-1661.

De Natale G., Troise C., Mark D., Mormone A., Piochi M., Di Vito M.A., Isaia R., Carlino S., Barra D., Somma R. (2016) The Campi Flegrei Deep Drilling Project (CFDDP): New insight on caldera structure, evolution and hazard implications for the Naples area. Geochemistry, Geophysics, Geosystems, 17, 4836-4847. 


\section{EnPreŝs}

De Vita S., Orsi G., Civetta L., Carandente A., D’Antonio M., Deino A., di Cesare T., Di Vito M.A., Fisher R.V., Isaia R., Marotta E., Necco A., Ort M., Pappalardo L., Piochi M., Southon J. (1999) The Agnano-Monte Spina eruption 4100 years BP in the restless Campi Flegrei caldera, Italy. Journal of Volcanology and Geothermal Research, 91, 269-301.

Di Fiore V., Aiello G., Beranzoli L., Bortoluzzi G., Castellano M., D’Anna G., Favali P., Marsella E., Patanè D. (2006) Studio di utilizzazione di sorgenti sismiche marine tipo Airgun nell'esplorazione sismica tomografica di vulcani attivi: l'esempio del Vulcano Stromboli. Extended Abstract, GNGTS 2006, pp. 167-170.

Knott C.G. (1899) Reflection and refraction of elastic waves with seismological applications. Philosophical Magazine, 48, 64-97.

Judenherc S., Zollo A. (2004), The Bay of Naples (southern Italy): Constraints on the volcanic structures inferred from a dense seismic survey. J. Geophys. Res., 109, B10312, doi:10.1029/2003JB002876.

ISPRA (2017) Secondo rapporto sugli effetti per l'ecosistema marino della tecnica dell'airgun. Ministero dell'Ambiente, Roma, Italia, pp. 1-33.

Landmark Graphic Corporation (1998) Promax 2D Seismic Processing and Analysis. User Manual, pp. 1-386, Texas, U.S.A.

Lomax A., Zollo A., Capuano P., Virieux J. (2001) Precise absolute earthquake location under Somma-Vesuvius volcano using a new three-dimensional velocity model. Geophysical Journal International, 146, 313-331.

Liu G., Fomel S. (2013) Seismic data analysis using local time-frequency decomposition. Geophysical Prospecting, 61 (3), 516-525.

Liu G., Fomel S., Chen X. (2011) Time-frequency analysis of seismic data using local attributes. Geophysics, 76 (6), P23-P34.

Marr J.D., Zagst E.F. (1967) Exploration horizons from new seismic concepts of CDP and digital processing. Geophysics, 32, 207-224.

Mayne W.H. (1962) Horizontal data stacking techniques. Supplement to Geophysics, 27, 927-938.

Mayne W.H. (1967) Practical considerations in the use of common reflection point techniques. Geophysics, 32, 225-229.

Mormone A., Troise C., Piochi M., Balassone G., Joachimski M., De Natale G. (2015) Mineralogical, geochemical and isotopic features of tuff from CFDDP $506 \mathrm{~m}$ hole: Hydrothermal activity in the eastern side of the Campi Flegrei volcano (Southern Italy). Journal of Volcanology and Geothermal Research, 290, 39-52.

Pescatore T.S., Diplomatico G., Senatore M.R., Tramutoli M., Mirabile L. (1984) Contributi allo studio del Golfo di Pozzuoli - Aspetti stratigrafici e strutturali. Memorie della Società Geologica Italiana, 27, 139-149.

Scarpa R., Bianco F., Del Pezzo E., Tronca F., La Rocca M. (2000) Shallow velocity structure and heterogeneity of Mt. Vesuvius, Italy, from seismic array analysis. In: Capuano P., Gasparini P., Zollo A., Virieux J., Casale R., Yeroyanni M. (Eds.) The Internal Structure of Mt. Vesuvius, pp. 183-202.

Scarpati C., Perrotta A., Lepore S., Calvert A. (2013) Eruptive history of Neapolitan volcanoes: Constraints from ${ }^{40} \mathrm{Ar} /{ }^{39} \mathrm{Ar}$ dating. Geological Magazine, 150, 412-425.

Sheriff R.E., Geldart R.P. (1995) Exploration Seismology. Cambridge University Press., $2^{\text {nd }}$ Edition, pp. 1-419. 


\section{EnPress}

Torrente M.M., Milia A. (2018) Comment on "The Campi Flegrei Deep Drilling Project (CFDDP): New insight on caldera structure, evolution and hazard implications for the Naples area (Southern Italy)" by G. De Natale et al. Geochem., Geophys. Geosystems.

Xu S., Zhang Y., Pham D., Lambarè G. (2005) Antileakage Fourier transform for seismic data regularization. Geophysics, 70 (4), V87-V95.

Yilmaz O. (1987) Seismic data processing. Society of Exploration Geophysics, Tulsa.

Wang P., Gao J., Wang Z. (2014) Time-Frequency Analysis of Seismic Data Using Synchrosqueezing Transform. IEEE Geoscience and Remote Sensing Letters, 11 (12), December 2014.

Zoeppritz K. (1919) On the reflection and penetration of seismic waves through unstable layers. Goettinger Nachr, 66-84.

Zollo A., Gasparini P., Virieux J., Biella G., Boschi E., Capuano P., De Franco R., Dell'Aversana P., De Matteis R., De Natale G., Iannaccone G., Guerra I., Le Meur H., Mirabile L. (1998) An image of Mt. Vesuvius obtained by 2D seismic tomography. Journal of Volcanology and Geothermal Research, 82, 161-173.

Zollo A., D'Auria L., De Matteis R., Herrero A., Virieux J., Gasparini P. (2002) Bayesian estimation of 2-D P-velocity models from active seismic arrival time data: imaging of the shallow structure of Mt.Vesuvius (Southern Italy). Geophysical Journal International, 151, 566-582.

Zollo A., Judenherc S., Auger E., D’Auria L., Virieux J., Capuano P., Chiarabba C., de Franco R., Makris J., Michelini A., Musacchio G. (2003) Evidence for the buried rim of Campi Flegrei caldera from 3-d active seismic imaging. Geophysical Research Letters, 30(19), 2002, doi:10.1029/2003GL018173, 2003.

Zollo, A., Maercklin N., Vassallo M., Dello Iacono D., Virieux J., Gasparini P. (2008), Seismic reflections reveal a massive melt layer feeding Campi Flegrei caldera. Geophysical Research Letters, 35, L12306, doi: 10.1029/2008GL034242. 


\section{Tables}

\begin{tabular}{|l|l|}
\hline Type of source & n.2 GI Gun SI/Sodera -210 cubic inch \\
\hline Length of the seismogram & $5 \mathrm{sec}$ \\
\hline Sampling interval & $1 \mathrm{msec}$ \\
\hline Distance among the sources & $25 \mathrm{~m}$ \\
\hline Distance among the hydrophones & $12.5 \mathrm{~m}$ \\
\hline
\end{tabular}

Table 1: Seismic acquisition parameters.

\begin{tabular}{|l|l|}
\hline Processing Workflow & \\
\hline 1. Geometry assignment & Field data \\
\hline 2. Trace editing & \\
\hline 3. Parameter selection & \\
\hline 4a. Elevation Statics & \\
\hline 4b. Refraction Statics & Pick First Breaks \\
\hline 5. Brute Stack & \\
\hline 6. Velocity analysis & \\
\hline 7. Residual statics & \\
\hline 8. Dip Moveout (DMO) & \\
\hline 9. Post-stack Signal Enhancement & Velocity modeling \\
\hline 10. Post-stack Migration & \\
\hline
\end{tabular}

Table 2: General workflow of seismic data processing with Promax 2D.

\begin{tabular}{|l|l|}
\hline Data Compression & \\
\hline $\begin{array}{l}\text { Discrete Cosine Transform } \\
\text { dctcomp Compression by Discrete Cosine Transform }\end{array}$ & \\
dctuncomp Discrete Cosine Transform Uncompression & \\
\hline Packing & \\
supack1 Pack segy trace data into chars & \\
supack2 Pack segy trace data into 2 byte shorts & \\
suunpack2 Unpack segy trace data from shorts to floats & \\
\hline $\begin{array}{l}\text { Wavelet Transforms } \\
\text { wpc1 comp2 Compress a 2D seismic section trace-by-trace }\end{array}$ & \\
\hline
\end{tabular}




\section{EnPress}

\section{using Wavelet Packets}

wpcluncomp2 Uncompress a 2D seismic section, which has been compressed using Wavelet Packets

wpccompress Compress a 2D section using Wavelet

Packets

wpcuncompress Uncompress a 2D section

wptcomp Compression by Wavelet Packet Compression

wptuncomp Uncompress WPT compressed data

wtcomp Compression by Wavelet Transform

wtuncomp Uncompression of WT compressed data

Table 3: General workflow of seismic data compression with Seismic Unix.

Editing, Sorting and Manipulation

Edit + Tools

suabshw Replace header key word by its absolute value

suazimuth Compute trace AZIMUTH given the sx,sy,gx,gy header fields and set a user-specified header field to this value

subset Select a SUBSET of the samples from a 3-dimensional file

suchw Change Header Word using one or two header word fields

sucountkey Count the number of unique values for a given keyword

suedit Examine segy diskfiles and edit headers

sugethw Sugethw writes the values of the selected key words

sukill Zero out traces

sunan remove NaNs \& Infs from the input stream

suquantile display some quantiles or ranks of a data set

surange get max and min values for non-zero header entries

sushw Set one or more Header Words using trace number, mod and integer divide to compute the header word values or input the header word values from a file

sutab print non zero header values and data for non-graphic terminals

suwind window traces by key word

suxedit examine segy diskfiles and edit headers

\section{Sort}

susort sort on any segy header keywords

susorty make a small 2-D common shot off-end data set in which the data show geometry

values to help visualize data sorting 


\section{EnPress}

\section{Manipulate}

fcat fast cat with 1 read per file

maxdiff find absolute maximum difference in two segy data sets

segyhdrmod replace the text header on a SEGY file

suaddnoise add noise to traces

sucmp CoMPare two seismic data sets, returns 0 to the shell if the same and 1 if different sudiff,susum,suprod,suquo difference, sum, product, quotient of two SU data sets via suop2

suflip flip a data set in various ways

suhtmath do unary arithmetic operation on segy traces with headers values

suinterp interpolate traces using automatic event picking

sumixgathers mix two gathers

sunull create null (all zeroes) traces

suop do unary arithmetic operation on segys

suop2 do a binary operation on two data sets

supermute permute or transpose a $3 \mathrm{~d}$ datacube

suramp Linearly taper the start and/or end of traces to zero

surecip sum opposing offsets in prepared data

recip sum opposing (reciprocal) offsets in cdp sorted data

suresamp Resample in time

resamp Resample the 1st dimension of a 2-dimensional function $\mathrm{f}(\mathrm{x} 1, \mathrm{x} 2)$

suswapbytes Swap the bytes in SU data to convert data from big endian to little endian byte order, and vice versa

sutaper Taper the edge traces of a data panel to zero

sutxtaper Taper in $(\mathrm{X}, \mathrm{T})$ the edges of a data panel to zero

suvcat append one data set to another, with or without an overlapping region. Data in the overlap may be determined by one of several methods

suzero zero-out data within a time window

swapbytes Swap the bytes of various data type

transp Transpose an $n 1$ by $n 2$ element matrix

Table 3: General workflow of editing, sorting and manipulation with Seismic Unix. 
Figures

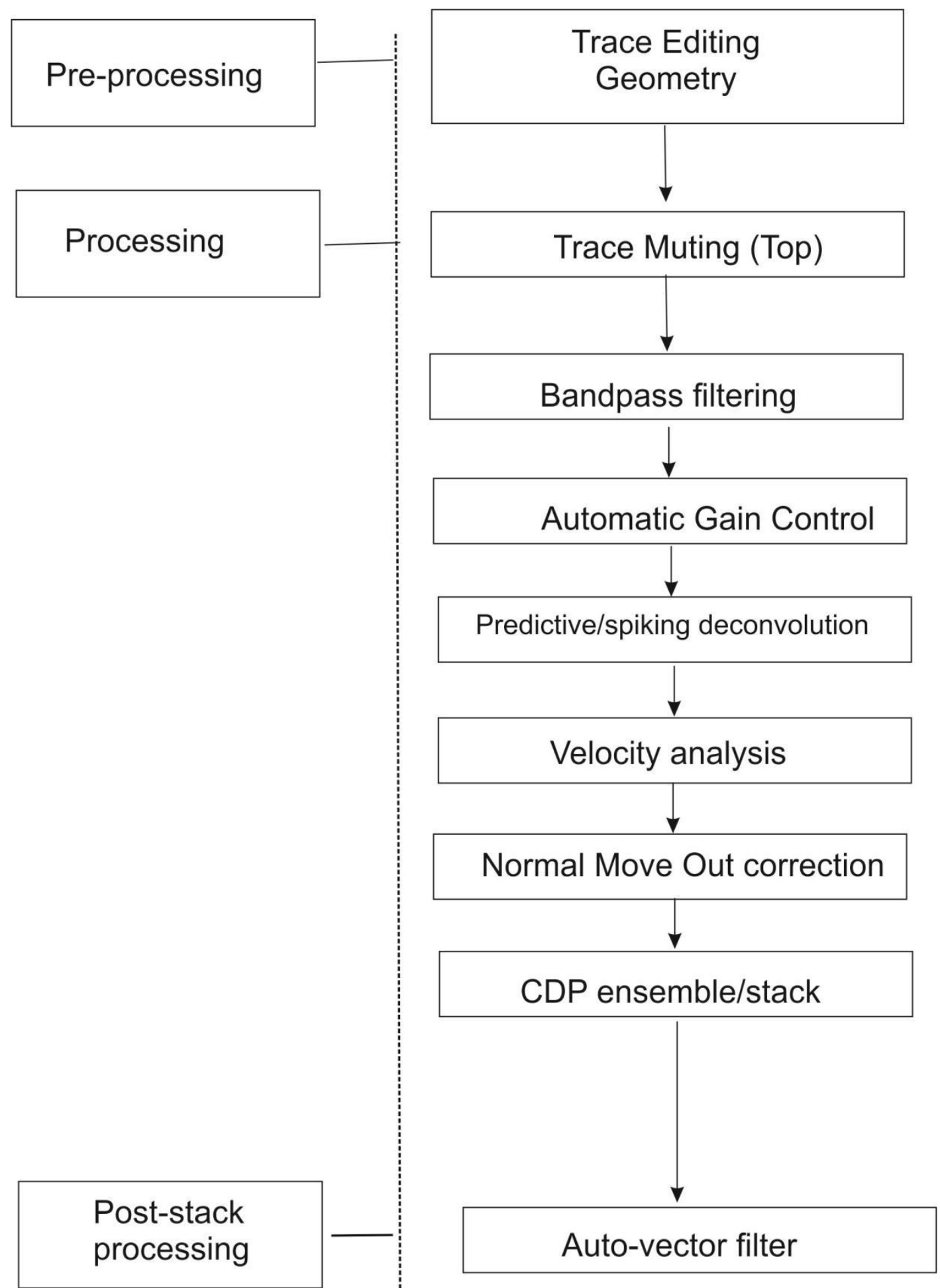

Fig. 1: Flux of elaboration of seismic data. 


\section{$\langle\hat{\mathrm{e}}\rangle \mid$ EnPresss}
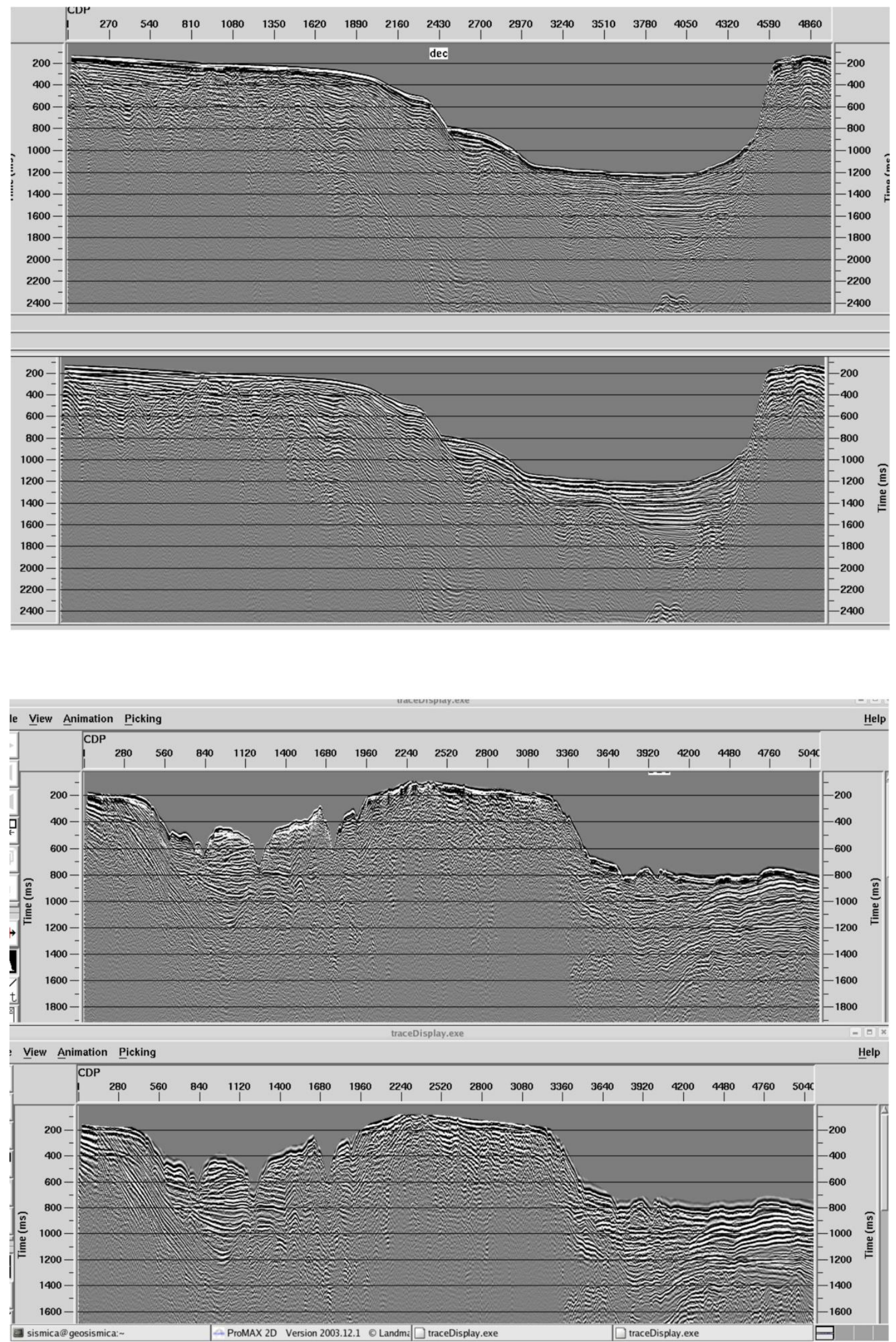

Fig. 2: Application of deconvolution to the seismic profiles of the Gulf of Naples. 


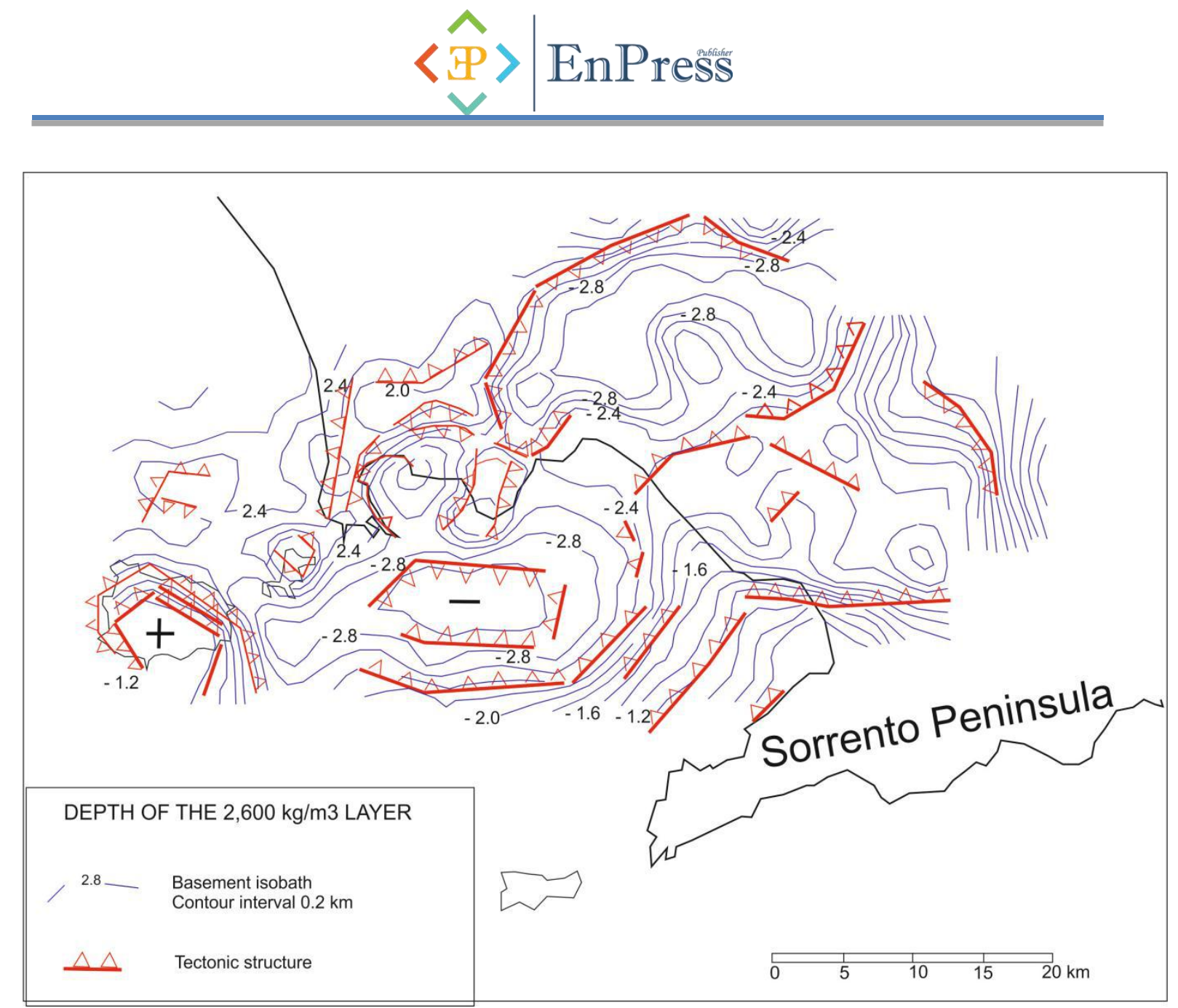

Fig. 3: Structural map of the carbonate basement in the Gulf of Naples. 